J3eA - Vol. 3 - 1 (2004).

DOI : 10.1051/bib-j3ea:2004001

\title{
Approche inductive d'un problème de régulation concret (Apprendre par l'action)
}

\author{
C.-P. Rauch * (École des Mines de Nantes) \\ Mis en ligne le 30/01/2004.
}

\begin{abstract}
Résumé
De nombreuses raisons incitent désormais le monde enseignant à revoir ses méthodes de travail. Afin d'illustrer l'emploi des méthodes actives d'apprentissage, l'article décrit un module de développement de compétences spécifiques dans le champ des sciences physiques. Basée sur l'assemblage et la découverte inductive d'un dispositif de régulation de température, la méthode privilégie l'implication personnelle de l'étudiant à toutes les étapes.
\end{abstract}

Les étudiants réalisent, testent et maîtrisent personnellement tous les dispositifs constituants de l'expérience, avant de les assembler en un système dont ils découvrent empiriquement le comportement. L'enseignant les aide ensuite à bâtir leur propre modèle mathématique de ce comportement. Seules l'observation et la confrontation à la réalité permettent de valider ou non le modèle : l'étudiant prend alors conscience de la distance entre sa représentation et la réalité.

Le résultat est une pédagogie exigeante pour l'étudiant comme pour l'enseignant, mais aussi un plus dans la formation initiale de l'ingénieur.

Mots-clés : pédagogie active, éducation nouvelle, apprentissage par problèmes, apprentissage par l'action, réactivité, sciences physiques, régulation thermique.

(C) EDP Sciences, 2004.

Niveau de connaissances requis. Mathématiques : équations différentielles linéaires du premier ordre. Électrocinétique du niveau de première année de DEUG ou de classe préparatoire.

Niveau des étudiants. Élèves-ingénieurs de niveau $\mathrm{Bac}+2$ ou $\mathrm{Bac}+3$.

* Carl-Philippe Rauch ${ }^{1}$, ingénieur ENSMA 1982, a obtenu un DEA Optique et Traitement de l'image en 1989. Il est enseignant-chercheur en sciences physiques. Depuis 1995, son activité est centrée sur la formation des élèves-ingénieurs par voie expérimentale : travaux pratiques et projets expérimentaux, puis apprentissage par l'action. Il développe une méthodologie de formation complémentaire aux formes traditionnelles, destinée à promouvoir un véritable esprit scientifique et répondre aux besoins actuels, et pratique simultanément l'accompagnement scientifique à l'école élémentaire. Il milite pour le décloisonnement des niveaux d'instruction et des domaines d'apprentissage.

o e-mail : Carl.Rauch@emn.fr

${ }^{1}$ École des Mines de Nantes, BP 20722, F-44307 Nantes Cedex 3, France. 


\section{Introduction}

Aux niveaux Bac +2 et $\mathrm{Bac}+3$, sous l'appellation d'Apprentissage par l'Action (Apa), l'Ecole des Mines de Nantes développe et met en oeuvre depuis cinq ans à l'échelle de promotions complètes des méthodes actives d'enseignement des sciences, dans le but d'améliorer l'efficacité globale du parcours de formation proposé aux étudiants. Après un survol des méthodes actives d'apprentissage et des principes de la méthode Apa, cet article présente une version du module de sciences physiques adaptée au profil d'une vingtaine d'étudiants qui intègrent l'École au niveau Bac+3.

\section{L'enseignement scientifique}

\subsection{Un contexte mouvant}

À l'image des profondes mutations en cours dans la société, les besoins en matière de formation initiale - pour n'aborder qu'elle - évoluent régulièrement, alors que les méthodes de formation employées sur le terrain restent majoritairement traditionnelles. Il s'ensuit inévitablement un décalage.

La jeunesse aborde actuellement les formations post-baccalauréat avec un profil inédit : culture générale, savoirs, aptitudes conceptuelles, aspirations, qualités humaines prennent des dimensions nouvelles - favorables ou inquiétantes -. Les jeunes supportent de moins en moins l'approche frontale et abstraite des sciences, comme semble le prouver leur désaffection actuellement constatée pour les cursus scientifiques.

À l'autre extrémité du parcours de formation, les attentes du monde professionnel portent de plus en plus sur les compétences réelles et l'aptitude à gérer les changements, à apprendre par soi-même et à oeuvrer en groupe [1].

Dans ce contexte mouvant, une question de fond se pose : la place même des sciences fondamentales dans la formation n'est-elle pas à redéfinir ? En l'absence de réponse pertinente, on peut au moins stimuler l'intérêt des étudiants et accroître l'efficacité de l'enseignement scientifique en diversifiant l'approche des sciences offerte.

\subsection{Les méthodes actives}

Leur objectif est d'aider l'étudiant à découvrir de nouveaux phénomènes ou concepts par lui-même, à relier ceuxci à ses connaissances antérieures et à structurer son intelligence globale du domaine.

Malgré leur niveau d'abstraction, les études supérieures posent, sur le plan pédagogique, les mêmes difficultés de principe que l'école élémentaire. Concernant les méthodes actives, il peut s'avérer utile d'établir un parallèle entre ces deux antipodes de la formation scientifique.

\subsection{1. À l'école élémentaire}

La fin du XIX ${ }^{\text {ème }}$ siècle a vu l'émergence d'un courant de contestation de la pédagogie classique, accusée d'étouffer la créativité en empêchant l'enfant de construire par lui-même ses savoirs et compétences [2].

De nos jours, l'opération Main à la Pâte, lancée en 1996 par le Prix Nobel de physique Georges Charpak pour donner un nouvel élan à l'enseignement scientifique à l'école élémentaire [3], s'inscrit dans les principes de l'éducation nouvelle ainsi définie un siècle plus tôt : mise en situation, démarche expérimentale inductive, importance de l'écrit et de la confrontation citoyenne, sont autant de principes qui conduisent l'enseignant à reconsidérer son rôle et son action pour ne plus brider l'envie de comprendre des enfants.

Sur un plan pratique, en Loire-Atlantique, l'École des Mines de Nantes a démontré que l'implication d'un établissement scientifique dans l'accompagnement des enseignants est extrêmement favorable à une réforme en profondeur des méthodes d'apprentissage scientifique à l'école. 


\subsubsection{Dans l'enseignement supérieur}

La méthode dite des situations-problèmes semble d'usage courant dans les écoles de commerce. Apparu au Canada, l'Apprentissage par Problèmes (en anglais $\mathrm{Pbl}$ : Problem-based learning) s'est aussi développé depuis quelques décennies, surtout en médecine et en droit (ex : Université John Hopkins, Université de Maastricht, Université des Andes à Bogota). Par groupes, les étudiants sont amenés à résoudre par eux-mêmes, sans apport théorique préalable, des problèmes concrets de difficulté croissante aptes à leur inculquer le noyau de savoirs essentiels à la matière étudiée [4]. Il est regrettable que globalement peu de formations soient dispensées par cette méthode en sciences et techniques [5] : bien que lourde à mettre en oeuvre pour l'enseignant, elle semble bien mener à une acquisition plus durable des savoirs par l'étudiant.

D'autres formes se développent actuellement, telles que la désormais classique pédagogie par projet [6] et l'Apa. L'intérêt grandissant du monde enseignant pour ces formes de pédagogie suscite d'ailleurs actuellement la création d'une communauté internationale consacrée au développement de la pédagogie active [7].

\subsection{L'Apprentissage par l'action (Apa)}

L'Apa, dérivé pour sa version de sciences physiques d'un cours conçu au Caltech de Pasadena par le professeur Jerry Pine, est décliné à Nantes, d'une part en mathématiques (analyse, probabilités) pour l'acquisition d'un programme de connaissances précis, et d'autre part en sciences physiques [8] (voici un « spectacle» maintenant habituel dans les couloirs de l'École).

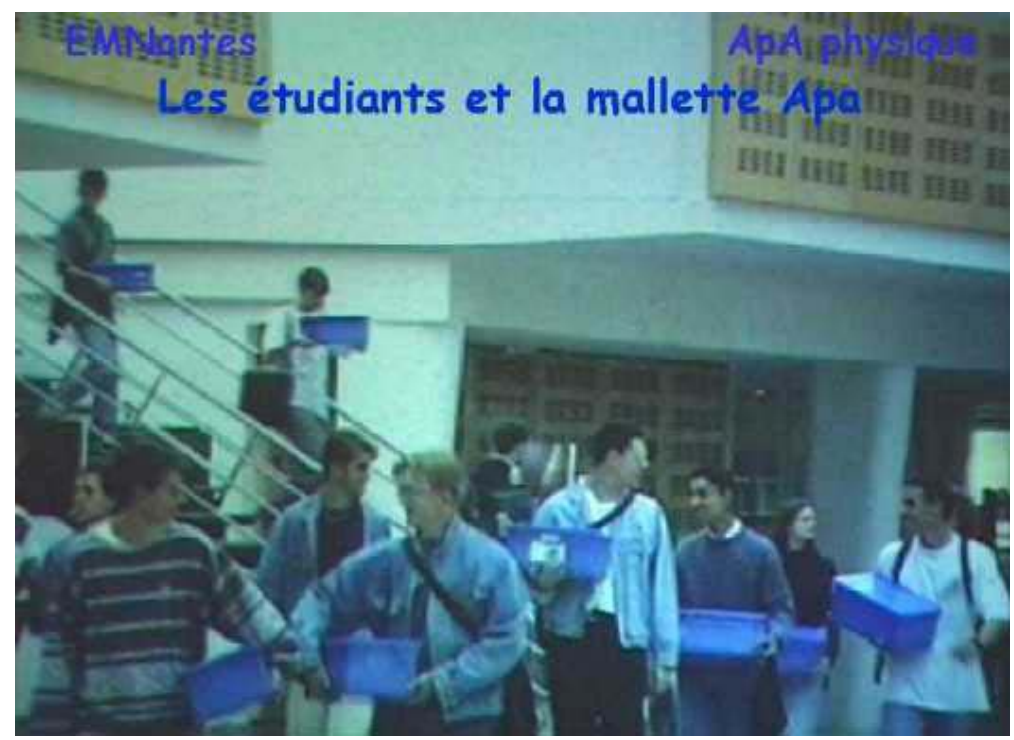

Fig. 1. Les étudiants et la mallette Apa.

Dans ce dernier cas, l'objectif n'est pas l'acquisition de nouvelles connaissances théoriques, mais celui de stimuler, via l'investigation scientifique, la réactivité des étudiants et réconcilier avec la physique, c'est-à-dire développer un corpus de compétences :

- leur capacité d'analyse des systèmes physiques et du monde réel,

- des méthodes de travail adaptées à la résolution effective de problèmes concrets,

et les sensibiliser au caractère incertain et complexe des situations réelles [9].

Au bout de six mois, le module de physique enseigné à $\mathrm{Bac}+2$ conduit les étudiants à concevoir et produire librement leur propre expérience de mesure ou de conversion d'énergie. À ce stade, seule compte la démarche : les moyens disponibles limitent la performance, mais ce facteur contextuel n'est pas du ressort de l'étudiant.

Le module à $\mathrm{Bac}+3$ décrit ci-dessous se focalise, quant à lui, sur un montage élémentaire de régulation de température dans une enceinte. 


\section{Découverte inductive d'un processus de rélation}

\subsection{Chronologie}

Chaque étudiant dispose d'une mallette de matériel (transformateur, multimètre, composants électroniques et pièces mécaniques) et d'outillage (fer à souder, outils) qui contient tout le nécessaire à la réalisation individuelle et progressive de la base technique du module. Dans un premier temps, ces 4 circuits électroniques (alimentation basse tension -BT-, capteur de température, amplificateurs de tension et de puissance, associés à divers montages expérimentaux tous faits main, font l'objet d'étapes de caractérisation : mesure du taux de régulation en charge de l'alimentation, étalonnage du capteur pour la mesure absolue de températures, réglage du gain de l'amplificateur de tension, validation de l'amplificateur de puissance. Primordiales pour la suite, ces étapes doivent être menées à bien par chaque étudiant et posent bien des problèmes à certains ! La seconde partie du module porte sur la découverte du dispositif de régulation et se déroule en binômes.

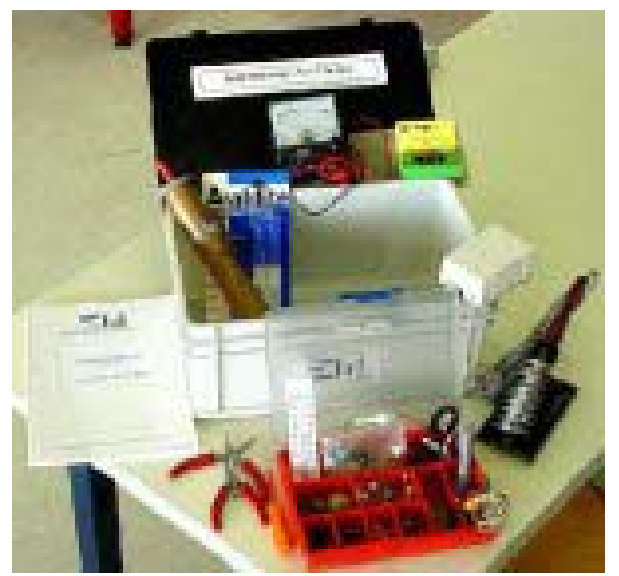

Fig. 2. Une mallette Apa standard.

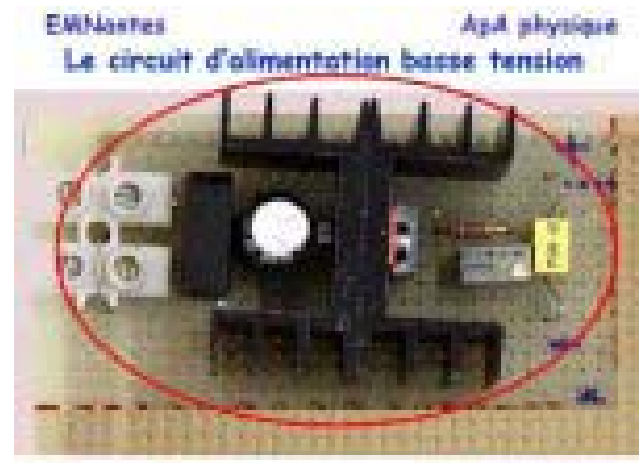

Fig. 3. Le circuit d'alimentation BT.

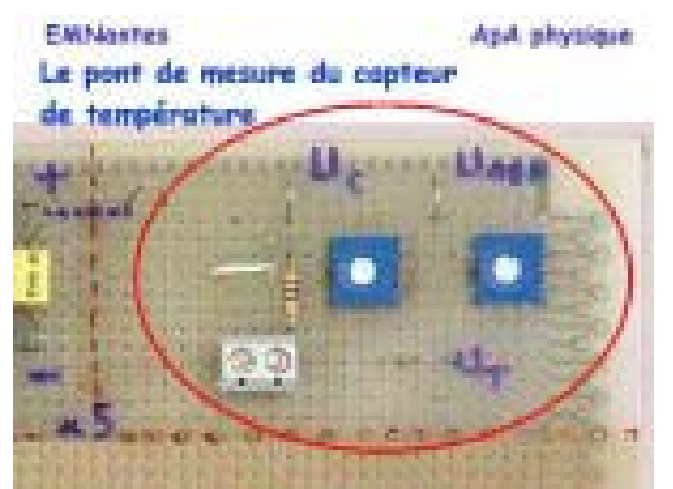

Fig. 4. Le pont de mesure du capteur de température. 


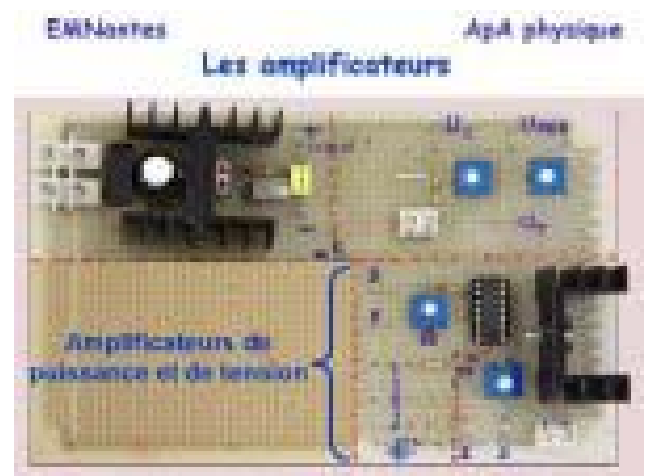

Fig. 5. Les circuits amplificateurs et l'ensemble des circuits développés par chaque étudiant.

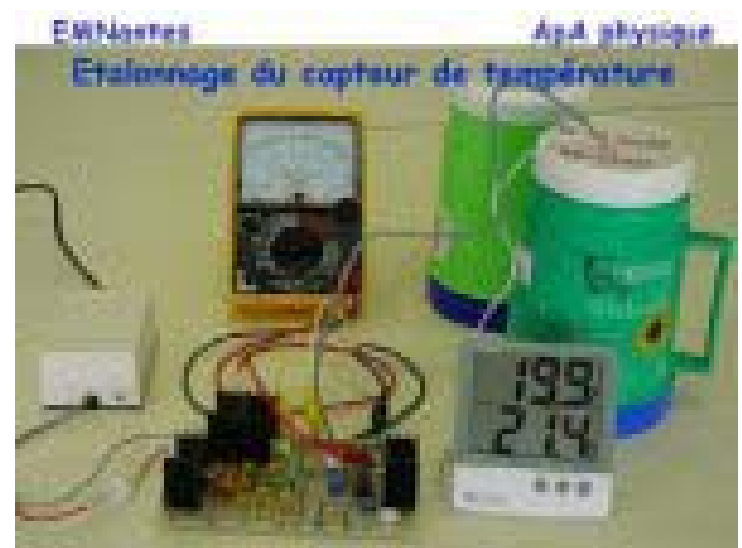

Fig. 6. Une procédure techniquement peu coûteuse pour l'étalonnage du capteur de température.

\subsection{Organisation générale (Fig. 7)}

L'essentiel du travail de préparation est effectué librement à domicile, selon un plan de charge défini par l'enseignant. Des séances en classe régulières permettent le suivi et la validation des résultats, ainsi que les activités d'investigation décrites ci-dessous. Chaque thème abordé est structuré en trois temps, correspondant à un niveau croissant de conceptualisation :

- la résolution des problèmes techniques (schémas, soudage, pannes, réglages);

- la mise en situation pour une découverte par chacun de l'expérience ; l'élaboration collective ou guidée des nouveaux concepts, outils ou méthodes nécessaires ; la poursuite de l'expérience qui doit être menée à son terme par chaque binôme ;

- la rédaction d'un rapport de synthèse en temps limité, sur la base des données personnelles compilées au préalable (documents, fiches d'expérience, ...).

Si les deux premiers temps sont clairement des temps d'apprentissage (cliquer ici pour en savoir plus sur les objectifs et les contenus d'apprentissage), le premier n'en est pas moins soumis à des contraintes de délais fortes (obligation de résultats). 
Quelques telments diorparisation

- 40 ta d Ferplei du tempt zur 10 aenainet

- Au fotal, 60 h de trakeil pour Ifthudiant

- Mallette indhiduelle prittie por IfEcole

- Uh eneignont pour 18 a 22 itudientr

- Fraluation der acquis:

- trowil de spothise individuel peiriadique

- cumitale rtgulier du repeet des delaia at des ohjectifs

Fig. 7. Quelques éléments d'organisation générale.

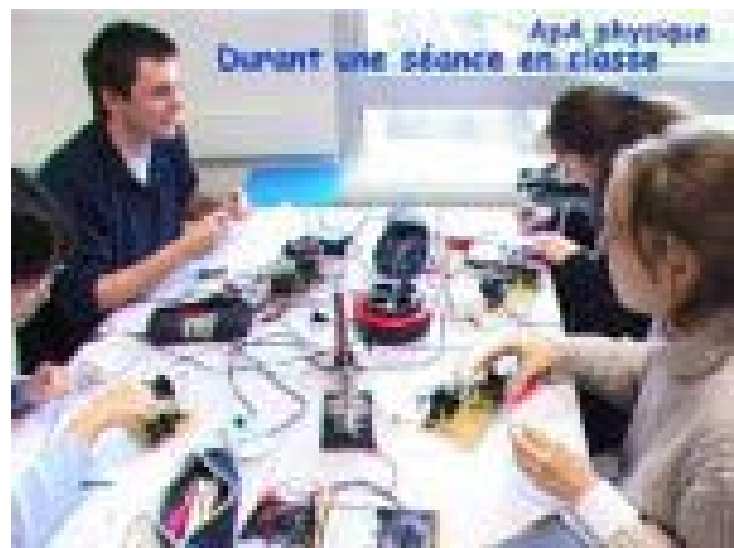

Fig. 8. L'activité durant une séance en classe.

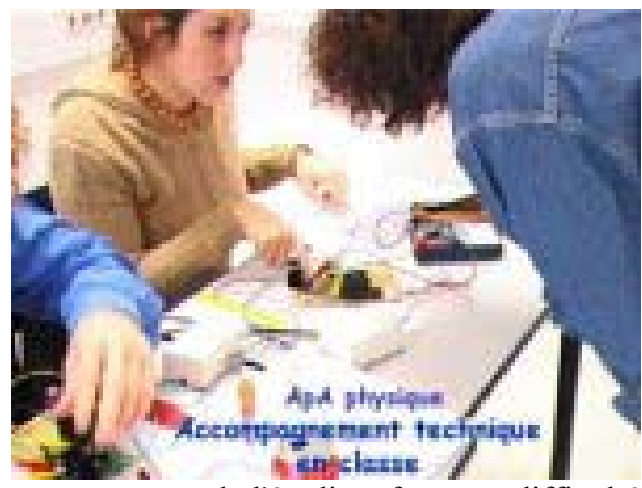

Fig. 9. L'accompagnement de l'étudiant face aux difficultés techniques.

Euptastes:

Apd physipt

Objectift depprentissegt

" Accuirl he mithater at nuab-faine de linerstiget on reiertifiqu

" Ar rendre trombins a noi-mine les ponnalmanem arcundifep de longer date

- Divelepper un Níl aprit reimsifizur : pregnatime. averturs, ertativits

= Et peut-Etre, à cette occerian, retrourer kr gout des selences in.

Fig. 10. Synthèse des objectifs d'apprentissage. 


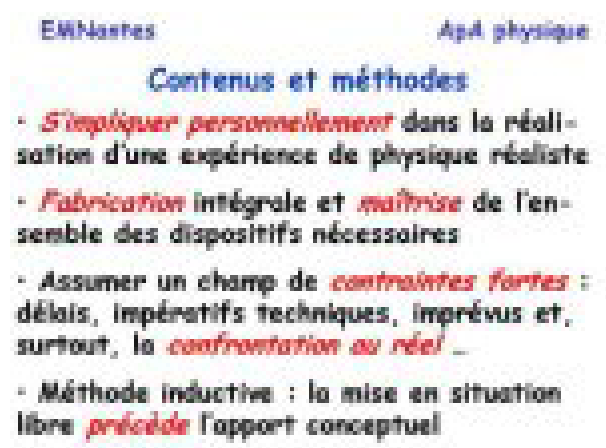

Fig. 11. Les contenus et méthodes mis en oeuvre pour atteindre les objectifs.

\section{3. Étude de la boucle de régulation}

Le dispositif doit maintenir à une valeur de consigne de $35^{\circ} \mathrm{C}$ la température interne d'un « four » fait d'un gobelet plastique équipé d'une résistance de chauffage (lampe) et d'une thermistance. L'objet de l'étude est de construire, sur la base du comportement observable du dispositif, un modèle mathématique capable de reconstituer ce comportement aussi bien que possible. Savoir modéliser efficacement le réel est, en effet, présenté aux étudiants comme la base de la constitution d'un outil prédictif, l'une des compétences fondamentales de l'ingénieur.

\subsubsection{Le montage}

Chaque binôme dispose (si tout s'est bien passé auparavant!) de 2 ensembles complets de circuits opérationnels : un étudiant peut donc fournir le capteur de température, constitué de la thermistance Th et d'un pont de mesure, l'autre peut mettre à disposition la partie correction et puissance.

[Nota - un point de technique : pour des raisons de conflit de potentiel électrique, il est indispensable d'alimenter le capteur par l'intermédiaire d'une alimentation BT distincte de l'alimentation des amplificateurs. D'où la nécessité de faire travailler les étudiants en binômes].

Le signal de retour $\mathrm{U}(\mathrm{t})$, image de l'écart de température du four par rapport à la consigne, a la propriété de varier en raison inverse de la température.

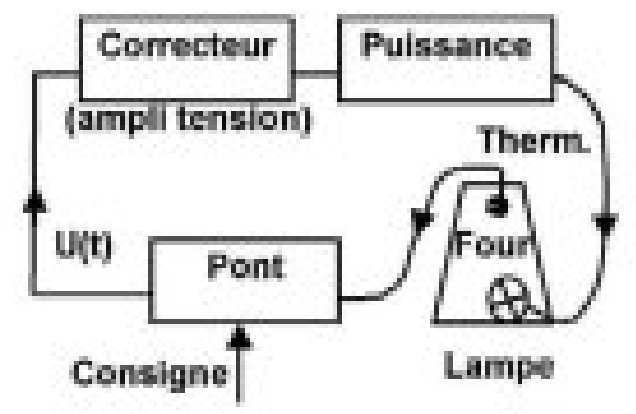

Fig. 12. Schéma de principe du dispositif d'expérience. 


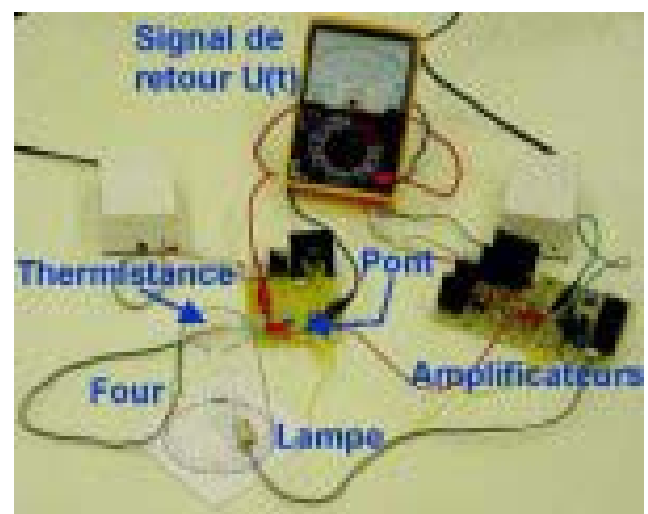

Fig. 13. Vue d'ensemble du montage.

\subsubsection{Démarche d'investigation}

- Première approche

Les étudiants doivent imaginer et faire fonctionner un circuit assurant la fonction cherchée. Après une période de tâtonnement, ils parviennent au montage ci-dessus (un montage élémentaire de régulation proportionnelle), mais s'étonnent de découvrir qu'il subsiste toujours, en régime stabilisé, un écart résiduel par rapport à la consigne.

\section{- Recherche d'un modèle}

Pour construire un modèle mathématique du dispositif, ils étudient le comportement du four seul. Sur les conseils de l'enseignant, ils procèdent à l'identification en boucle ouverte (réponse à un échelon de commande, le four étant assimilé à un système dynamique du $1^{\mathrm{er}}$ ordre) et en déduisent l'équation différentielle régissant la boucle fermée en réponse à un échelon de consigne.

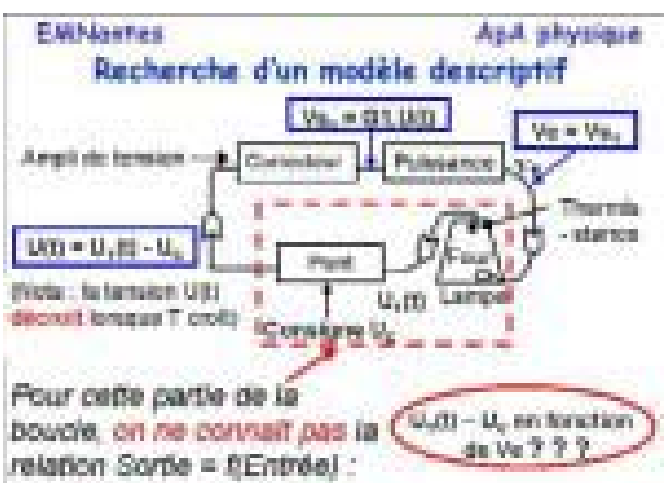

Fig. 14. Élaboration d'un modèle mathématique du comportement de la boucle de régulation.

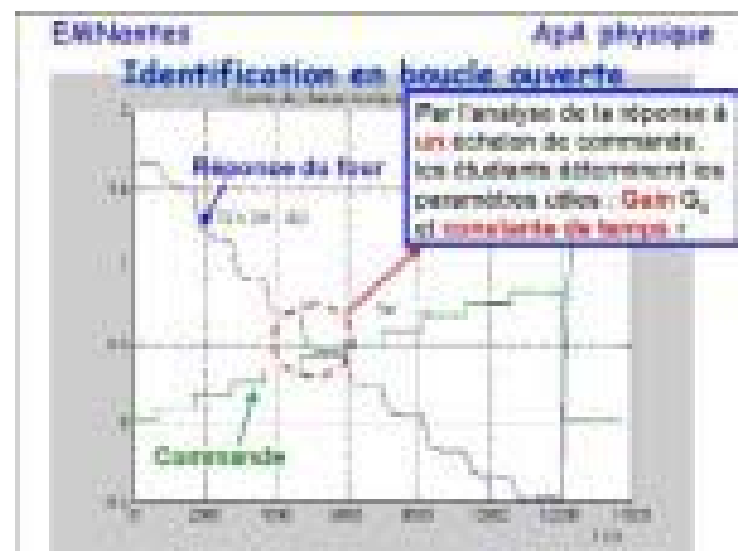

Fig. 15. La procédure d'identification en boucle ouverte des paramètres caractéristiques du système [four + capteur de température]. 


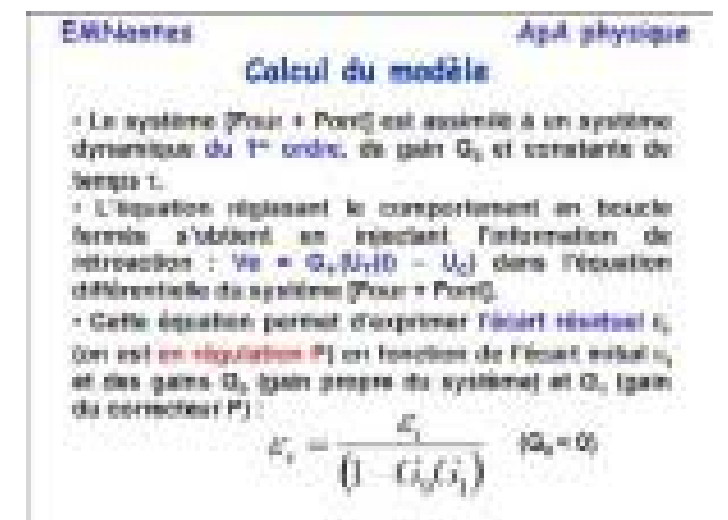

Fig. 16. Principe de la modélisation du système en boucle fermée.

- Résultats

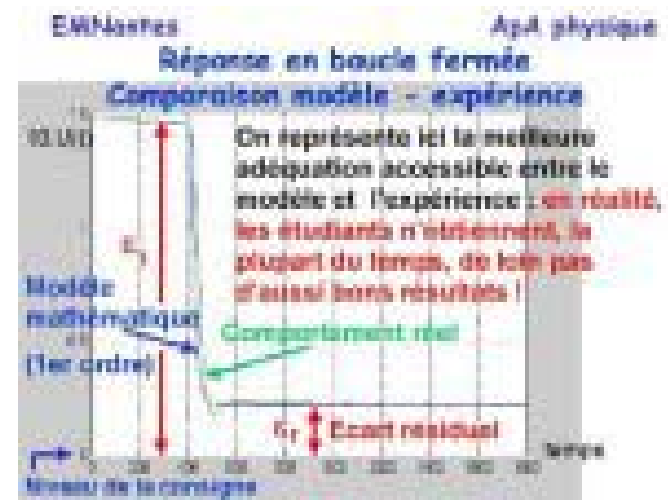

Fig. 17. Courbe de réponse en boucle fermée.

Les courbes présentées ci-dessus résultent d'une démarche plus élaborée que celle présentée précédemment (voir ci-dessous : « Affiner le modèle »). À l'issue d'une démarche menée sans raffinement ni précaution particulière, la courbe de comportement théorique et la courbe de réponse expérimentale des étudiants diffèrent parfois notablement, la différence étant particulièrement nette quant à l'écart résiduel (voir la figure précédente pour la définition de l'écart résiduel).

\section{- Affiner le modèle}

Pourquoi de telles différences entre expérience et modèle ? Sans aide de l'enseignant, les étudiants ne perçoivent pas que les variations de la température extérieure influent sur les paramètres propres du four : le système est non-linéaire, ses caractéristiques dépendent de l'écart entre la température interne du four et la température ambiante. Une fois avertis, les étudiants procèdent à une nouvelle identification de ces paramètres dans de bonnes conditions expérimentales : la comparaison théorie - expérience est alors tout à fait satisfaisante, conforme aux courbes ci-dessus. 


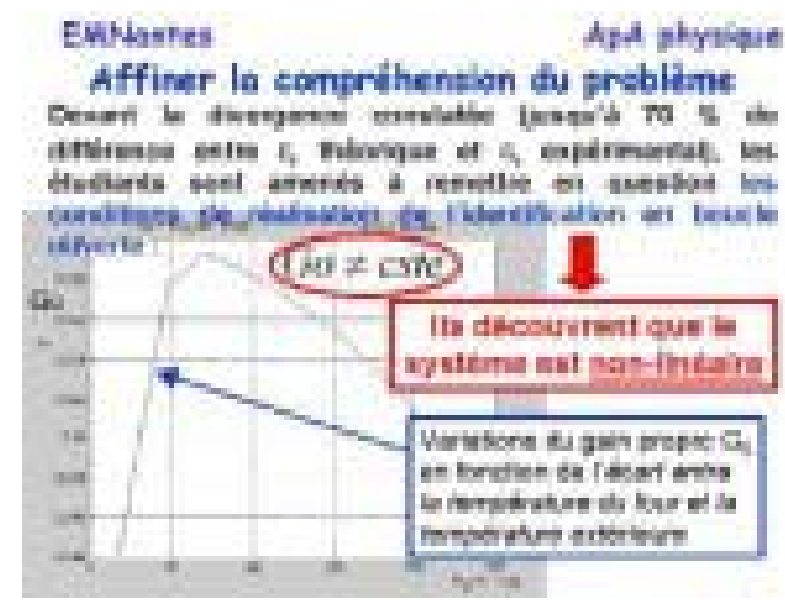

Fig. 18. Prendre conscience de la nécessité d'affiner la modélisation du système.

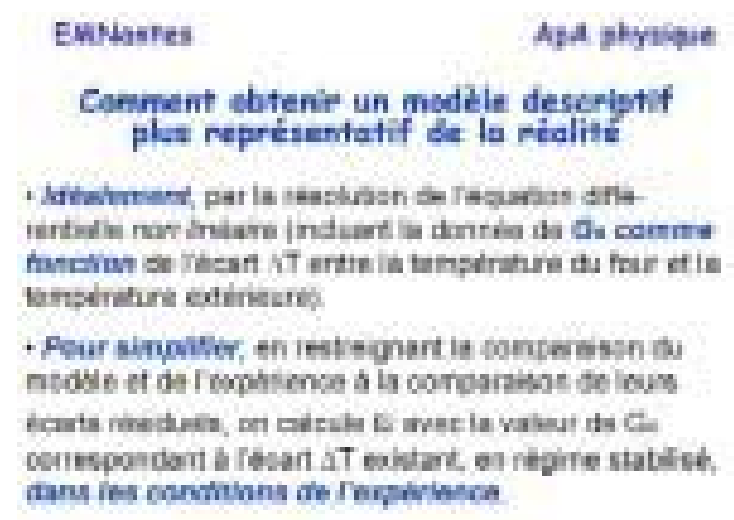

Fig. 19. Comment parvenir à un modèle mathématique plus représentatif de la réalité.

\section{- Passage en correction PI}

Par une transformation élémentaire de l'amplificateur de tension en amplificateur-intégrateur, les étudiants constatent que la température rejoint la consigne en régime stabilisé, selon un régime transitoire oscillant ou amorti selon le condensateur utilisé.

L'extension du modèle théorique précédent à ce cas montre que le modèle n'est pas à même de décrire correctement le comportement de la boucle lorsque celui-ci évolue rapidement (cas des faibles constantes de temps d'intégration).

- Pour aller plus loin

Un nouveau raffinement du modèle peut être entrepris, par une prise en compte plus précise du comportement du four en boucle ouverte (premier ordre avec retard ou deuxième ordre).

\subsection{L'évaluation}

Des démonstrations, réglages, enregistrements de courbes sur PC ou mesures directes de paramètres par l'enseignant permettent de valider régulièrement les résultats techniques obtenus par chaque étudiant ou binôme. L'évaluation des méthodes de travail est aussi fort nécessaire : l'étudiant s'est souvent formé seul, comme il le pouvait, sur cette compétence primordiale. Enfin, des travaux de synthèse personnels permettent d'évaluer l'assimilation des sujets scientifiques étudiés.

Induire la réflexion, conseiller sans déflorer le sujet ni imposer trop tôt son avis, évaluer au bon moment mais pas en permanence... : la mission de l'enseignant est donc complexe. 


\section{Conclusion}

L'exigence est forte envers les enseignants, qui accompagnent les étudiants, font bien plus que leur dispenser un savoir et assument au quotidien les nombreux imprévus techniques.

Pour les étudiants issus de classe préparatoire, cette pédagogie est nouvelle : soumis à un champ de contraintes fortes, ils apprennent à affronter par eux-mêmes des situations inconfortables, des insuccès, doivent assumer leurs limites mais découvrent aussi une nouvelle manière de faire de la physique et l'apprécient pour la plupart.

\section{Références bibliographiques}

[1] R. Germinet, L'apprentissage de l'incertain (Éditions Odile Jacob, 1997).

[2] M. Fournier, Un siècle d'éducation nouvelle, Sciences Humaines, 105 (2000) 44-46.

[3] Site officiel de la Main à la Pâte : http://www.inrp.fr/lamap (consulté le 20 janvier 2004).

[4] Pour une présentation de la méthode : F. Hunot-Clairefond, Former les nouveaux managers (Liaisons, 1996).

[5]Ex : cours de statistiques de $2^{\mathrm{e}}$ année à l'École des Mines de Douai ; à l'étranger : cours d'automatique de $3^{\mathrm{e}}$ année à l'Université des Andes (Bogota).

[6] G. Lachiver et D. Dalle, Les défis de l'apprentissage par problèmes et par projets en ingénierie : l'exemple de l'université de Sherbrooke, Actes du $2^{\mathrm{e}}$ colloque « Questions de pédagogie dans l'enseignement supérieur », ENSIETA/ENST Bretagne, Brest (France), juin 2003.

[7] $2^{\text {nd }}$ workshop on active learning in engineering education, Copenhague (Danemark) / Göteborg (Suède), 3-7 juin 2002 ; http://www.ale2002.dtv.dk (consulté le 20 janvier 2004). Pour l'année $2004: 4^{\text {th }}$ workshop on active learning in engineering education, Nantes (France), 6-9 juin 2004 ; http://www.ale-net.org (consulté le 20 janvier 2004).

[8] J. Miller-Jones et al., Active learning in engineering education, actes IMICET, Insa de Lyon (France), 6-9 juin 2001.

[9] C. Rauch, L'apprentissage par l'action en sciences physiques, une forme différente et complémentaire des formes traditionnelles d'enseignement, Actes du 2ème colloque « Questions de pédagogie dans l'enseignement supérieur », ENSIETA/ENST Bretagne, Brest (France), juin 2003, 259-264.

Pour en savoir plus sur les développements de la pédagogie par l'action à l'École des Mines de Nantes, voir la référence :

[10] S. Tabiou, P-B. Gossiaux et L. Bot, Une expérience pédagogique d'enseignement des mathématiques en école d'ingénieurs, Actes du $2^{\mathrm{e}}$ colloque « Questions de pédagogie dans l'enseignement supérieur », ENSIETA/ENST Bretagne, Brest (France), juin 2003, 67-71. 